\section{1}

\title{
A Synthetic Biology Approach to Sequential Stripe Patterning and Somitogenesis
}

\author{
Fuqing Wu, ${ }^{1 \dagger}$ Changhan He, ${ }^{2}$ Xin Fang, ${ }^{3}$ Javier Baez, ${ }^{2}$ Thai Ohnmacht, ${ }^{4 \ddagger}$ Qi Zhang, ${ }^{1}$ Xingwen \\ Chen, ${ }^{1}$ Kyle R. Allison, ${ }^{3}$ Yang Kuang, ${ }^{2}$ Xiao Wang ${ }^{*}$
}

1School of Biological and Health Systems Engineering, Arizona State University, Tempe, AZ 85287, USA.

2School of Mathematical and Statistical Sciences, Arizona State University, Tempe, Arizona 85287, USA

3Wallace H. Coulter Department of Biomedical Engineering, Georgia Institute of Technology and Emory University; Division of Infectious Diseases, Department of Medicine, Emory University School of Medicine; Emory Antibiotic Resistance Center, Atlanta, Georgia, 30322, USA

${ }^{4}$ School of Life Sciences, Arizona State University, Tempe, AZ 85287, USA.

${ }^{\dagger}$ Current address: Department of Biological Engineering, Massachusetts Institute of Technology, 500 Technology Square, Cambridge, MA, 02139, USA.

₹Current address: Burrell College of Osteopathic Medicine, Las Cruces, New Mexico, 88001, USA

* Corresponding author:

Xiao Wang, Ph. D.

29 School of Biological and Health Systems Engineering,

30 Arizona State University, Tempe, AZ 85287, USA.

31 Tel: 1-480-727-8696

32 Fax: 1-480-727-7624

33 E-mail: xiaowang@asu.edu. 


\section{Abstract}

2 Reaction-diffusion (RD) based clock and wavefront model has long been proposed as the

3 mechanism underlying biological pattern formation of repeated and segmented structures

4 including somitogenesis. However, systematic molecular level understanding of the

5 mechanism remains elusive, largely due to the lack of suitable experimental systems to

6 probe RD quantitatively in vivo. Here we design a synthetic gene circuit that couples gene

7 expression regulation (reaction) with quorum sensing (diffusion) to guide bacterial cells

8 self-organizing into stripe patterns at both microscopic and colony scales. An

9 experimentally verified mathematical model confirms that these periodic spatial structures

10 are emerged from the integration of oscillatory gene expression as the molecular clock and

11 the outward expanding diffusions as the propagating wavefront. Furthermore, our paired

12 model-experiment data illustrate that the RD-based patterning is sensitive to initial

13 conditions and can be modulated by external inducers to generate diverse patterns,

14 including multiple-stripe pattern, target-like pattern and ring patterns with reversed

15 fluorescence. Powered by our synthetic biology setup, we also test different topologies of

16 gene networks and show that network motifs enabling robust oscillations are foundations

17 of sequential stripe pattern formation. These results verified close connections between

18 gene network topology and resulting RD driven pattern formation, offering an engineering

19 approach to help understand biological development.

\section{Main text}

24 Turing's seminal work first proposed reaction-diffusion (RD) as the "chemical basis of 25 morphogenesis" over six decades ago 1. It provides a general theoretical foundation of 26 pattern formation via RD mechanisms. Two decades later, RD driven clock and wavefront 27 (CW) mechanism was hypothesized as the mechanism underlying formation of repeated 28 and segmented structures such as somites in development ${ }^{2}$. Since then, although RD driven 29 pattern formation has been demonstrated or identified in chemical, physical, and ecological 30 systems ${ }^{3-10}$, its much-hypothesized role in multicellular pattern formation hasn't been fully 31 studied biologically. This is largely due to the lack of suitable model systems to test such

32 hypotheses. For example, somite development requires precise temporal and spatial 33 coordination between a heterogeneous web of intracellular responses and intercellular 
communications, both under control of complex gene regulation networks and influences of

2 universal gene expression stochasticity. Such complexity poses a great challenge to fully

3 understand mechanistic basis of somite formation in vivo. Engineered microbes carrying

4 rationally designed gene circuits provide an effective venue to study this problem from

5 bottom up. Previous studies using synthetic circuits have demonstrated formation of

6 predefined patterns, cell motility based stripe formation, and scale invariant ring pattern

7 formation 11-15. However, gene network directed RD based clock and wavefront pattern

8 formation, despite its importance in developmental biology and extensive theoretical

9 studies ${ }^{16-22}$, has not been experimentally realized.

11 Past studies have suggested that nonlinear multistable systems could also direct 12 spatiotemporal pattern formation when coupled with external diffusion process $23-25$. 13 Following this strategy to achieve a multicellular pattern formation, we designed and 14 constructed a mutually inhibitory network with positive autoregulation and 15 communications (MINPAC) by expanding our previously demonstrated quadrastable gene 16 circuit 26 with added quorum-sensing modules to enable intercellular communications (Fig. $171 \mathrm{~A}$ and $1 \mathrm{~B})$.

19 Specifically, the MINPAC topology is built upon two hybrid promoters Plas/tet and Plux/lac, 20 which harbor high nonlinearity and inducibility (Fig. 1A and Fig. S1). Plas/tet drives LasR, 21 LuxI and LacI expression, representing the node X in Fig. 1B, whereas Plux/lac regulates 22 transcription of LuxR, LasI, and TetR, representing the node Y. LasI and LuxI are synthases 23 that catalyze the synthesis of autoinducer 3-oxo-C12-HSL (C12) and 3-oxo-C6-HSL (C6), 24 respectively. The two small autoinducers can diffuse out of and into cells to mediate cell-cell 25 communication and coordinate population behaviors on a spatial domain. LasR and LuxR 26 activate Plas/tet and Plux/lac in the presence of C12 and C6, respectively, forming positive 27 autoregulations. IPTG inhibits the repressive effect of LacI on Plux/lac, and aTc counteracts 28 TetR inhibition on Plas/tet, forming the mutual inhibitions. Green fluorescent protein (GFP) 29 and mCherry protein serve as the corresponding reporters of Plux/lac and Plas/tet activities 30 in living cells (Fig. 1A). 


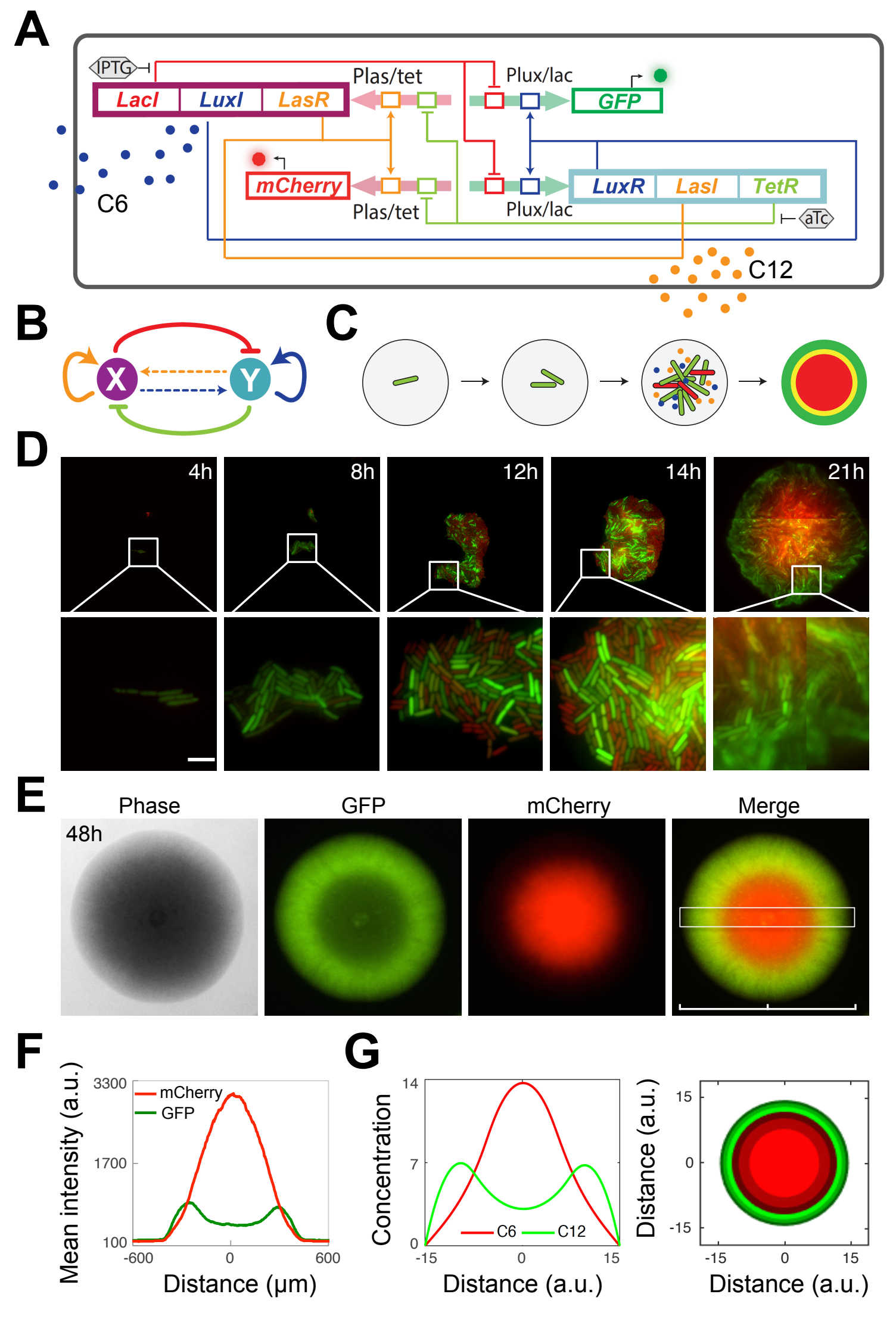


1 Fig. 1. Conceptual and experimental design of MINPAC, and reaction-diffusion based pattern formation. (A) Experimental design of the MINPAC network. Plas/tet (pink arrow) can be activated by LasR (yellow) and repressed by TetR (light green), while Plux/lac (green arrow) can be activated by LuxR (blue) and repressed by Lacl (red). Luxl (blue) synthesizes C6 (blue dots) to bind with LuxR to activate pLux/lac, while Lasl (yellow) synthesizes C12 (yellow dots) to bind with LasR to activate Plas/tet. GFP and mCherry serve as reporters for Plux/lac and Plas/tet. (B) Abstract diagram of MINPAC topology, where $X$ and $Y$ mutually inhibit each other ( $T$-bars) and auto-activate (arrowheads) itself, meanwhile $X$ and $Y$ can mutually activate through small autoinducer mediated intercellular communication (dashed arrowheads). Genes and regulations are color-coded corresponding to the circuit in (A). (C) Illustration of a ring pattern formation from a single $E$. coli cell harboring MINPAC circuit. (D) MINPAC directs single cells to self-organize into ring pattern at microscopic scale. Representative experiments of pattern formation from single cell to colony by time-lapse microscopy (Scale bar represents $5 \mu \mathrm{m}$ ). The 21 -hr image is captured and combined by four individual images. (E) MINPAC cells self-organized double-ring pattern at colony scale. Representative fluorescence images are taken at $48 \mathrm{hr}$. Magnification: 2x. (F) Mean fluorescence intensity across the center of pattern-generating colony (white box in $\mathbf{E}$ ). Distance indicates the size of the colony. (G) Left: PDE model simulations of the extracellular C6 and $\mathrm{C} 12$ concentrations, which are corresponding to mCherry and GFP intensities, respectively. Right: Two-dimensional ring pattern simulated from the model, with high C6 concentration (red) for cells in the core and high $\mathrm{C} 12$ concentration (green) on the edge of the colony, forming a similar double-ring pattern as in (E).

To investigate whether MINPAC could direct single cells to self-organize into spatial 25 patterns, we transformed the circuit into E. coli cells and serially diluted cell cultures into 26 single cells before seeding on a semi-solid minimal M9 medium (Fig. 1C). Using live singlecell time-lapse fluorescence microscopy, we observed the early stage of pattern formation (Fig. 1D). After an initial phase of uniform fluorescence (4 \& 8 hours), we observed that cells differentiated into equivalent numbers of green and red fluorescence in a disordered, seemingly-random, spatial distribution (12 \& 14 hours). As microcolonies grew to $\sim 100 \mu \mathrm{m}$ in diameter (between 14 and 21 hours of growth), a red-center green out-circle

32 fluorescence pattern starts to emerge (Fig. 1D). These results illustrate that our engineered 33 pattern formation is scale-dependent at the early stage and the pattern starts to emerge 34 only after cell number reaches a certain threshold. We reason that as the stochastic growth 35 progresses through time, while outcomes of cell-cell variability are hard to predict initially 
1 or at microscopic scale, the population starts to synchronize and converge to a collective

2 behavior and become more predictable as time progress or at macroscopic scale.

3

4 To further investigate the circuit's capability in directing pattern formation at macroscopic

5 scale, we carried out long term experiment by culturing single cell initiated colonies on agar

6 plates up to 96 hours. Time-lapse colony imaging results show that the single colony has no

7 obvious pattern at $15 \mathrm{hr}$ and exhibits a weak yellow flat disk, suggesting cells express either

8 GFP or mCherry are distributed without order (Fig. S2). This is consistent with our

9 microscopic observations. After $24 \mathrm{hr}$, cells in the colony started to differentially and

10 orderly express GFP and mCherry and self-organize into a stable double-ring pattern of an

11 outer GFP-ring and inner mCherry disk at $48 \mathrm{hr}$ (Fig. 1E and S2), with a small temporary

12 yellow ring between these two rings (Fig. S2). The double-ring pattern is stable with time.

13 Fluorescence quantification also confirms higher GFP expression for cells on the edge of the

14 colony and higher mCherry expression for cells in the center (Fig. 1F).

16 To rule out the possibility that circuit-independent factors such as nutrition or growth are

17 responsible for the pattern, we tested two control circuits: one with GFP and mCherry

18 expressed from constitutive promoters, and the other one with GFP and mCherry expressed

19 from hybrid promoters Plas/tet and Plux/lac. No obvious ring patterns were observed at 24

20 or 48 hrs (Fig. S3). Therefore, we conclude that MINPAC circuit is responsible for the self-

21 organized ring pattern in single colonies.

23 Toward a quantitative and mechanistic understanding of the ring patterning process, we 24 next built a partial differential equation (PDE) model to mathematically describe the 25 production, regulation, transport, and diffusion of autoinducers C6 and C12. LuxI and LasI's 26 expression in MINPAC governs the synthesis of C6 and C12, which can diffuse out of and 27 back into cells to further regulate the intrinsic transcriptional network MINPAC and 28 determine cells' fate spatially. Thus, the extracellular C6 and C12 kinetics serve as a 29 predictive snapshot of the spatial pattern and could represent the differential expression of 30 mCherry and GFP, respectively (see Supplemental materials for more details). Fitted with 31 biologically feasible parameters, our model shows the two autoinducers harbor similar 32 dynamics to experimental fluorescence intensities across the colony and can reproduce 33 experimentally observed ring pattern in two-dimensional geometry (Fig. 1G). Such 
1 corroboration between the RD-based PDE model and experimental results further verified

2 that observed ring pattern is the result of MINPAC regulated RD process.

A

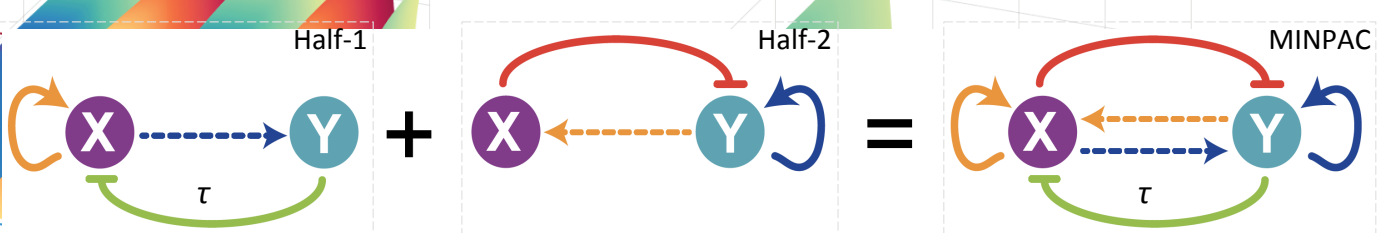

B

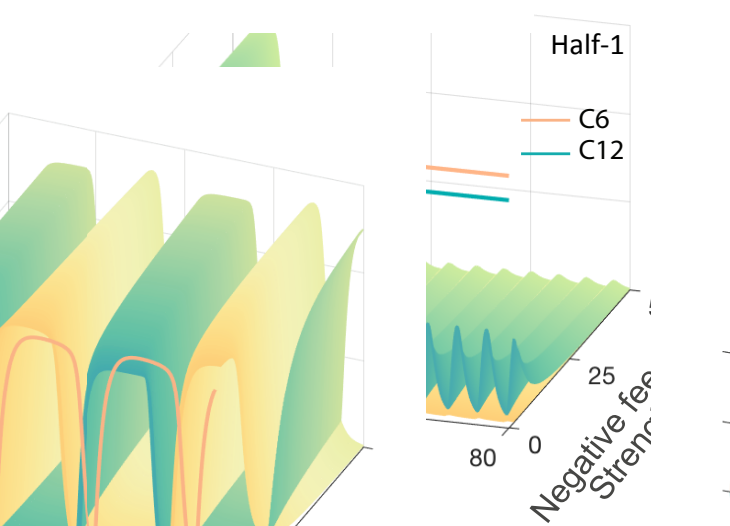

D

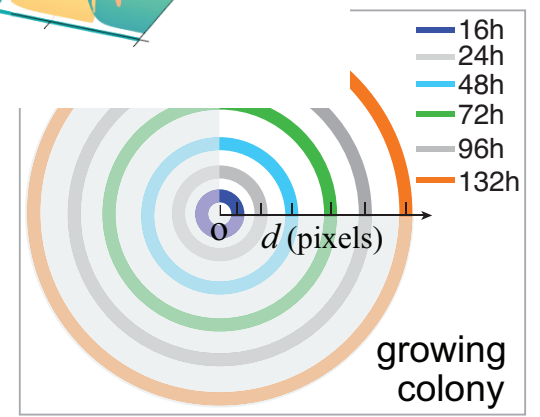

$\mathbf{F}$

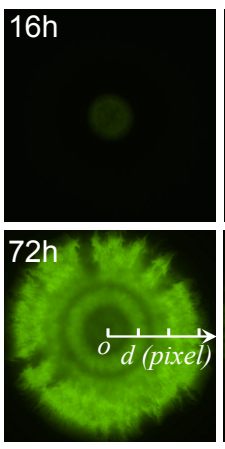

4
C

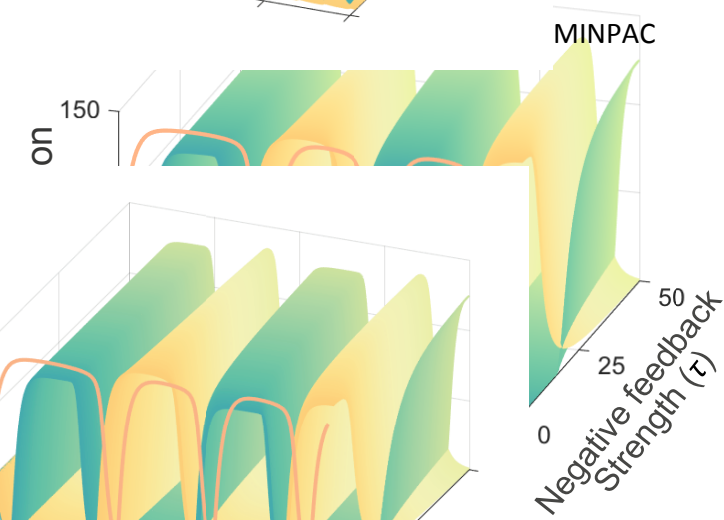

$\mathbf{E}$

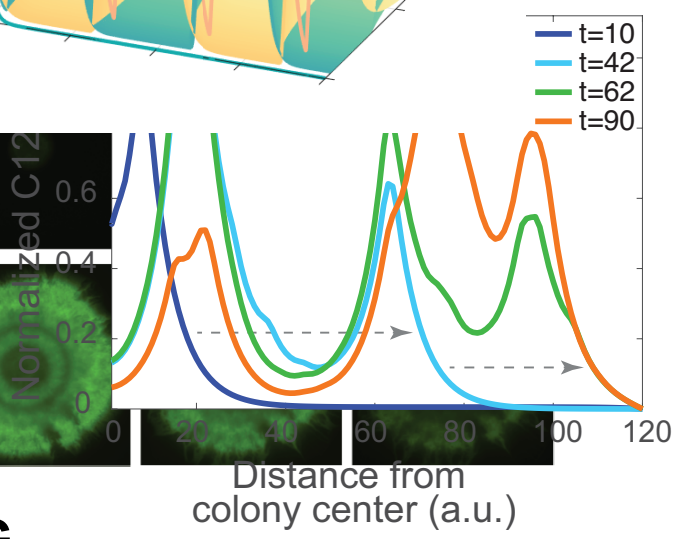

G

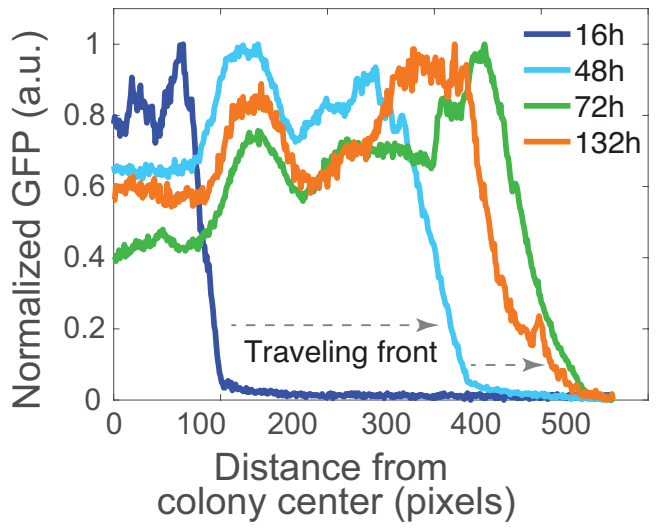


1 Fig. 2. MINPAC directs ring pattern formation through a reaction-diffusion based clock and wavefront mechanism. (A) Illustration of the MINPAC composition of two symmetric positiveplus-negative oscillator motifs. Parameter $T$ is used to describe the strength of one negative feedback (node $Y$ inhibits node $X$ ). (B-C) Model comparison between one-motif topology and twomotif MINPAC. Oscillation from one-motif topology is highly dependent on the parameter $T(\mathbf{B})$, whereas MINPAC harbors a greater robustness and amplitude against parameter $T$ changes to generate temporal oscillation (C). Cyan and yellow colormaps represent the C6 and C12 concentrations, respectively. The red and blue solid lines are C6 and C12 concentrations when $T$ equals to 0 (i.e. no negative feedback). (D) Diagram of a growing colony. Circles with different colors indicate the colony position at different time points. Center is labeled as $o$, and $d$ is the distance to the center of the colony. (E) Normalized external C12 concentration, directly correlated with experimental GFP intensities, of a pattern-growing colony with time and space from the PDE model simulation. Starting from the center of a colony, colored curves represent C12 concentrations along the colony radius at different time points. Grey arrows indicate the traveling direction of the wave front. (F) Time course of a growing colony having multiple GFP rings. (G) Quantified temporal and spatial fluorescence intensities of the multiple GFP ringforming colony in $(\mathbf{F})$, showing similar dynamics to model simulation in $(\mathbf{E})$. The distance starts from center of the colony from $16 \mathrm{hr}$ to $132 \mathrm{hr}$. Each pixel is $3.22 \mu \mathrm{m}$.

To further investigate how MINPAC directs the generation of ring pattern, we carried out deterministic analysis for the reaction term of the RD model (i.e. the ODE part). Time series shows that MINPAC has an oscillating reaction part (Fig. S4A), suggesting the temporal oscillation could drive an organized pattern formation across the expanding colony. From a network topology point of view, MINPAC is composed of two topologically equivalent motifs where a self-activating node activates the other node and it in turn inhibits the selfactivating node (Fig. 2A), each forming a robust positive-plus-negative oscillator topology 27-29. A fully symmetric MINPAC topology would rapidly go to stable steady states without oscillation, but little asymmetry between the two motifs would lead to a robust oscillation (Fig. S5). Our model-comparison results show that oscillation from one-motif topology is, as previously reported, highly dependent on the strength of its negative feedback $(\tau)$, which is

32 vital for cyclic gene expression27,30,31 (Fig. 2B). However, the two-motif MINPAC harbors a 33 greater robustness and amplitude against parameter perturbations to generate temporal 34 oscillation (Fig. 2C). Such robustness enhances the likelihood of observing our desired 35 phenotypic outputs from the synthetic gene circuit. 
A
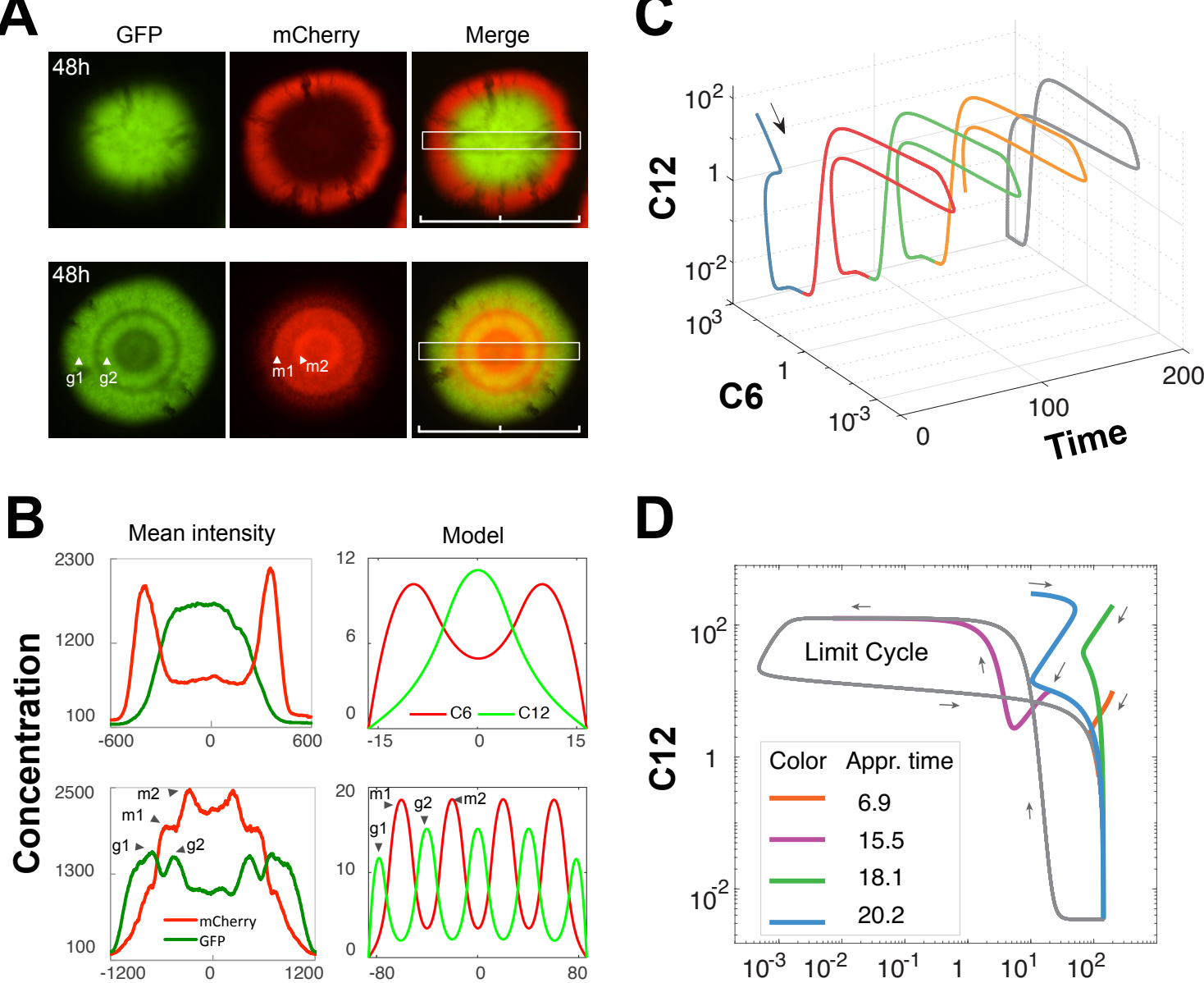

D

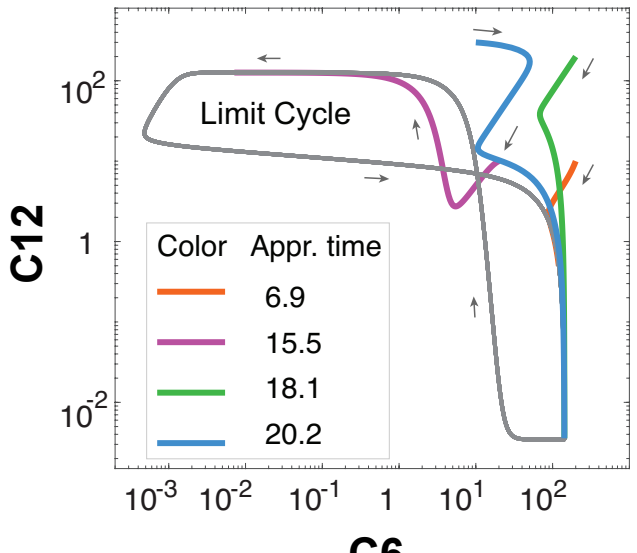

Fig. 3. Initial conditions and associated approaching time lead to diverse patterns. (A) Two observations distinct to Fig. 1E generated by MINPAC circuit. Top: a ring pattern with a GFP core and a mCherry outer ring; Bottom: a multiple GFP-mCherry ring pattern. (B) Left: Mean fluorescence intensities across the center of the ring-forming colonies in (A). Rings corresponding to the peaks are labeled. Right: Model simulations recapitulate experimental patterns only through changing the initial conditions of the model. (C) A trajectory of a random initial point (black arrow) going to oscillation periods (red, green and yellow curves) simulated from MINPAC reaction term. The grey "butterfly" curve illustrates the limit cycle. (D) Approaching time for different initial conditions. Colored curve shows the trajectory before stable oscillations and the approaching time is calculated for the solution going from its starting point to the stable limit cycle

13 (grey curve). 
1 In our MINPAC circuit, promoter functionality tests show LacI is less efficient to inhibit

2 promoter Plux/lac (Fig. S1A) compared to tetR to Plas/tet (Fig. S1B), supporting that the

3 asymmetric MINPAC could maintain an oscillatory gene expression profile as the molecular

4 clock. Moreover, the autoinducers' physical diffusion on the agar medium and colony

5 outward expansion (represented as one diffusion term in the PDE model) constitute the

6 propagating wavefront. Finally, the integration of clock and wavefront gates the engineered

7 bacterial cells into subgroups and segment spatially, generating periodic structures. This

8 reaction-diffusion based pattern formation is widely used to explain somitogenesis in

9 development $2,20,21$.

11 One interesting phenomenon among vertebrate species is the variations of somite numbers,

12 which is determined by the axis growth and presomitic mesoderm lifetime during 13 embryogenesis 32,33 . Analogously, we would expect multiple or even indefinite number of 14 stripes for a continuously growing colony (illustrated in Fig. 2D), and colonies with different 15 sizes would have different number of stripes when the oscillation frequency and colony16 expanding rates were constant across colonies. With our PDE model, we simulated the 17 temporal dynamics of C12 on the spatial scale and new peaks emerged periodically at the 18 wavefront (Fig. 2E, S4B). Experimentally, ring patterns with multiple stripes were also 19 observed sequentially by time lapse imaging of large colonies (Fig. 2F-G), as model 20 predicted. Collectively, these results suggest that the ring patterns we observed are the 21 outcomes of the spatiotemporal interaction of oscillatory dynamics owing to the network 22 topology and the movement stemming from the diffusion process.

24 However, even a macroscopic RD system could still be highly sensitive to initial conditions 25 due to the nonlinearity of the network interactions, evidenced by diverse patterns shown in 26 Fig. 3A, some colonies self-organize into a reversed double-ring pattern with GFP 27 accumulating in the inner ring and mCherry on the outer ring (top). A more complicated 28 pattern is also observed, in which two GFP rings alternating with two mCherry rings, 29 forming a multiple GFP-mCherry ring pattern (Fig. 2F and 3A, bottom). Given that these 30 different patterns emerge from the same MINPAC circuit operating in the same cells and 31 under the same conditions, we hypothesize that it is due to random variations of the initial 32 concentrations of intracellular proteins and autoinducers. To computationally test this 33 hypothesis, we tested various initial conditions of the PDE but kept all the parameters the 
1 same. The model indeed reproduces the experimental patterns (Fig. 3B). Furthermore,

2 these differences of the patterns suggest the system is not at steady state and, instead, is

3 evolving towards the steady state. The initial condition determines the starting point of the

4 MINPAC system, which will go through a temporal "non-oscillating" spiral (blue line in Fig.

5 3C) and finally approach oscillation periods (starting from red curve in Fig. 3C).

6 Quantitative simulations show that the oscillatory system, with different initial points, could

7 require significantly different times, so called Poincare return time, to approach the first

8 stable limit cycle (Fig. 3D). Thus, the initial condition and resulting approach-time variances

9 lead to diverse patterns with different stripes (besides colony size). These results illustrate

10 that initial conditions play an important role in shaping the formation of biological patterns,

11 which is consistent with recent theoretical analysis 16,34. Furthermore, the experiment-

12 model consistency entices us to use this model to analyze and predict newly emerged

13 patterns under different contexts.

15 To further examine the pattern's controllability, we next sought to apply external inducers

16 to perturb the regulations of MINPAC and hence pattern formation. C6, when applied

17 externally, would promote GFP expression and also LasI and TetR production, which could

18 both activate and inhibit mCherry expression. So the net impact of C6 induction is nonlinear

19 and nontrivial. Using the PDE model to simulate C6 application, it is predicted that we can

20 expect a multiple GFP-mCherry ring pattern when MINPAC is induced with external C6 (Fig.

214 A, top). Experimentally, we supplemented the medium with 1*10-8 M C6 and grow the

22 colony following the same protocol. Results show that the colony first formed an outer GFP

23 ring and a reddish yellow core at $24 \mathrm{hr}$, which became a red core at $60 \mathrm{hr}$ (Fig. S6A).

24 Strikingly, two GFP rings emerged at $77 \mathrm{hr}$ whereas mCherry mostly accumulated in the

25 center (Fig. 4B top, and Fig. S6A). Quantified fluorescence intensities also illustrate there are

26 four peaks for GFP and one significant peak for mCherry, which is in line with model

27 predictions (Fig. 4B). We noticed the inconsistent dynamics between predicted C6

28 concentrations and measured mCherry intensities, which is probably because of the slow

29 degradation rate of mCherry protein in living cells. Similarly, external C12 induction results

30 in two GFP rings with unbalanced intensities (Fig. S6B). 
A

B
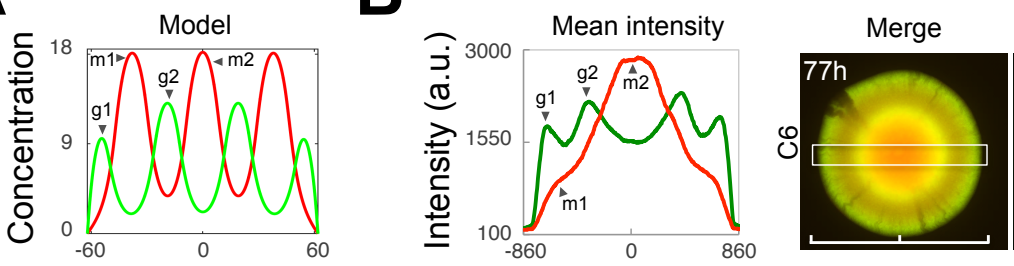

GFP
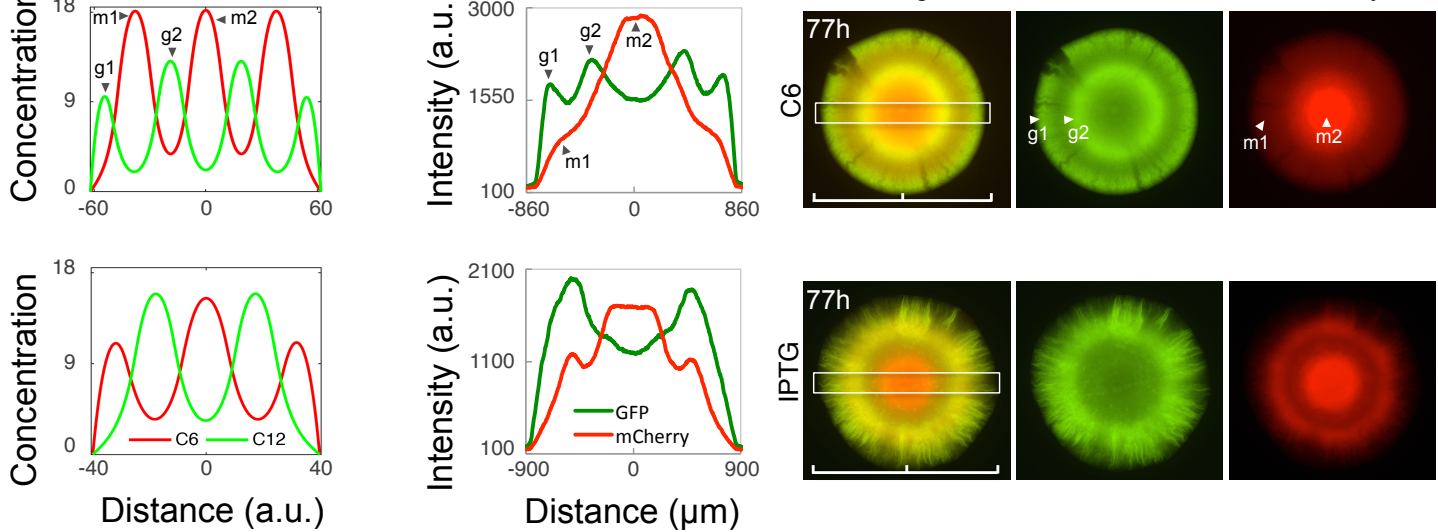

Distance (a.u.)

Distance $(\mu \mathrm{m})$

C
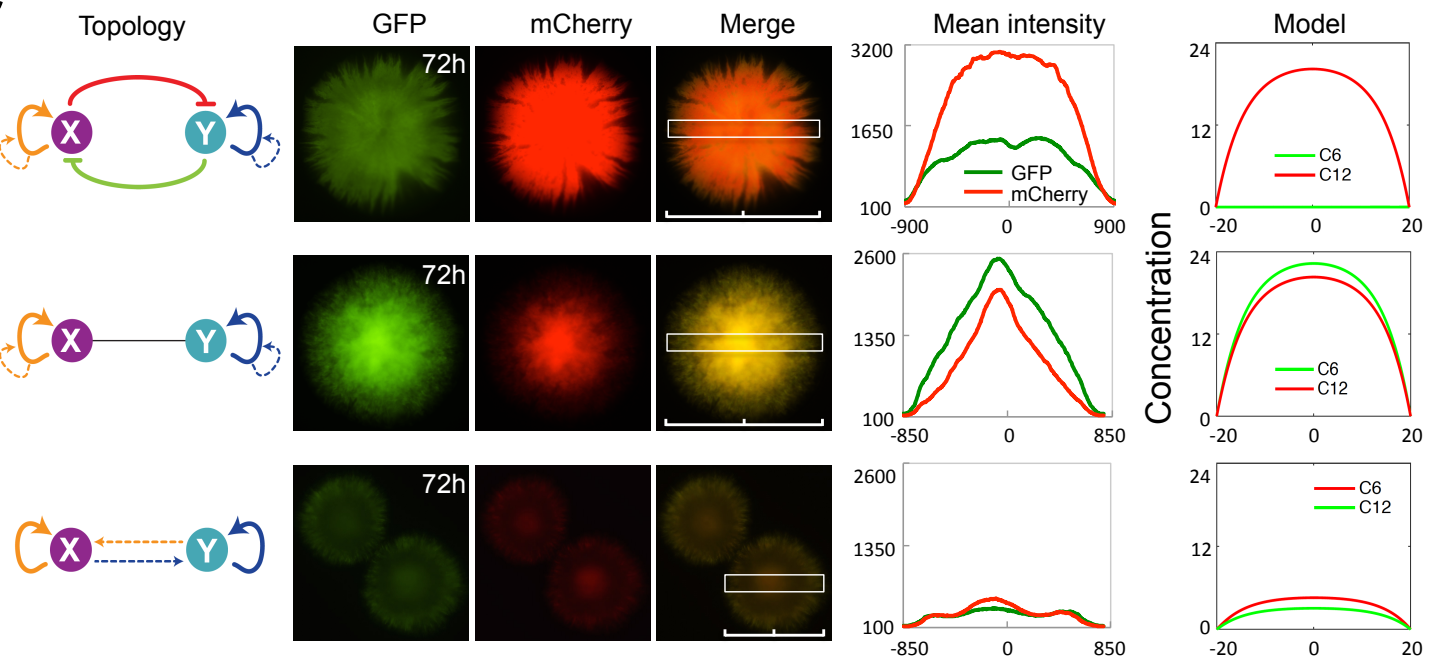

Distance $(\mu \mathrm{m})$

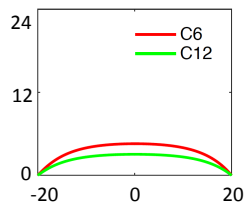

Distance (a.u.)

Fig. 4. MINPAC directed patterning is tunable and intrinsic to its network topology. (A) Model predictions of the pattern under external inducers C6 (top) and IPTG (bottom). (B) Experimental validations for model predictions, with C6 and IPTG induction. Top: two GFP rings were observed experimentally under $10^{-8} \mathrm{M} \mathrm{C6}$ induction at $77 \mathrm{hr}$. Its mean fluorescence intensity across the colony is similar to model prediction (A, top). Bottom: a target-like mCherry ring and an outer GFP ring were observed under $10 \mu \mathrm{M}$ IPTG induction. The mean fluorescence intensity is consistent to model prediction (A, bottom). Time course of pattern generation can be found in Fig. S6. (C) Three control circuits' topology and directed patterns. All the circuits are constructed with the same molecular components in MINAPC. Top left: A perturbed MINPAC topology. The intercellular $X-Y$ communications are replaced by intercellular auto-activation of $X$ and $Y$. No specific pattern is observed experimentally. Middle left: Mutual inhibition is removed and communication is replaced by intercellular auto-activation of $X$ and $Y$. Strong GFP and mCherry are simultaneously expressed and merged fluorescence is yellow. Bottom left: All regulatory 
edges are kept but the mutual inhibition module is removed. A weak yellow core and outer ring is observed. Middle: Mean fluorescence intensities across the center of the ring patterns. Right: Model simulations of the three control circuits show consistency to experimental results.

IPTG and aTc induction, on the other hand, can modulate the strength of mutual inhibition in the circuit. IPTG counteracts Lacl's inhibition on Plux/lac, leading to more LasI expression and intracellular C12 production. Simulating these changes by perturbing corresponding parameters, the model predicts a target-like mCherry ring with an outer GFP ring pattern (Fig. 4A, bottom), which is further verified by our experimental data (Fig. 4B, bottom). Time course shows that cells in the inner side of the GFP ring started to express mCherry, 12 showing as a yellow ring, at $\sim 60 \mathrm{hr}$ and was stable till $124 \mathrm{hr}$ (Fig. S6A). Inducer aTc's 13 impacts are similarly predicted and experimentally confirmed (Fig. S6C). Taken together, 14 these results illustrated the controllability of the MINPAC circuit and its directed patterns 15 formation. It is noteworthy that these patterns generated in single colonies autonomously without any predefined spatial cues and the regular structures are robust and stable once 17 formed.

Since the synthetic circuit directed cell-cell communication is established as a viable strategy to generate RD-based and tunable patterns, we employ this method to study the fundamental question of relationship between gene network topology and resulting multicellular pattern. We first designed a perturbed MINPAC topology, where the intercellular X-Y communication modules are replaced by intercellular auto-activations of $\mathrm{X}$ and Y (Fig. 4C, top, specific experimental design can be found in Fig. S7). Although there is still autoinducer diffusion, this circuit mitigates the interactions and dependency between $\mathrm{X}$ and $Y$ and would remarkably change the intrinsic dynamics. Both experimental observation and model simulation showed no specific pattern but a reddish colony (Fig. 4C, top row). Starting from this topology, we further removed the mutual inhibition module to construct a circuit with two positive feedback motifs (Fig. 4C, middle row), reinforced by intercellular activations. A yellow fluorescent colony with strong GFP and mCherry expression was observed, which is consistent with the model analysis. Lastly, we engineered a sub-network 32 of MINPAC, where the mutual inhibition is removed but keeping the other regulatory edges 33 (Fig. 4C, bottom row). Interestingly, this mutual-activation topology drives a weak yellow 34 target-like ring pattern with low GFP and mCherry expression (Fig. 4C, bottom row). 
1 Previous theoretical studies demonstrated that mutual-activation circuit with

2 autoregulations is multistable, and harbors a big parameter space for low-low state 35,36. Our

3 model analysis also confirms the low-GFP and low-mCherry expression in this sub-network

4 (Fig. 4C, bottom row). Taken together, each control circuit with different topology has

5 different fluorescence patterns but none of them show the alternating ring patterns,

6 indicating that the multiple-ring pattern is unique to MINPAC circuit.

8 Biological pattern formation requires complex gene regulation networks and accurate cell-

9 cell coordination. Indeed, coordinated cell population behavior in response to self-regulated

10 morphogen kinetics is a common phenomenon in development 8,37,38. Here, we present the

11 design and assembly of a synthetic gene network MINPAC, capable of directing engineered

12 single cells to form self-organized tunable patterns with multiple rings. The PDE model

13 simulations and experimental measurements strongly support that the observed ring

14 patterns are driven by a RD based oscillatory gene network with propagating wavefront, the

15 so-called clock and wavefront mechanism. It is noteworthy to point out that we used one

16 single PDE model to recapitulate and predict all the MINPAC-directed biological patterns.

17 Furthermore, we verified the close connections between gene network topology (circuit

18 architecture) and its induced spatial pattern formation.

20 MINPAC is a complete motif composed of intracellular transcriptional network and 21 intercellular communication modules, both of which cross-regulate each other to direct 22 spatial pattern formation involving the coordination of molecular gene expression, cellular 23 population response, and positional information interpretation. In this view, the MINPAC 24 represents a paradigm for future design of pattern-forming circuits. Moreover, similar 25 natural counterparts of MINPAC design can be found in the interaction networks of gap 26 genes for the anterior-posterior axis patterning in Drosophila ${ }^{39-41}$. Collectively, this work 27 provides a bottom-up synthetic biology approach to generate complex spatial patterns 28 arising from well-designed reaction-diffusion circuit motif, and integrates experimental 29 data with analytical framework across time and spatial scales to shed lights on the 30 molecular mechanisms of somitogenesis and biological pattern formation, which would 31 contribute to a better understanding of the natural developmental processes, and facilitate 32 the engineering of synthetic tissues in the future. 


\section{References}

4 1. Turing, A. M. The Chemical Basis of Morphogenesis. Philos. Trans. R. Soc. Lond. B. Biol. Sci. $5 \quad 237,37-72(1952)$.

6 2. Cooke, J. \& Zeeman, E. C. A clock and wavefront model for control of the number of 7 repeated structures during animal morphogenesis. J. Theor. Biol. 58, 455-476 (1976).

8 3. Lengyel, I. \& Epstein, I. R. A chemical approach to designing Turing patterns in reaction9 diffusion systems. Proc. Natl. Acad. Sci. U. S. A. 89, 3977-3979 (1992).

10 4. Maini, P. K., Painter, K. J. \& Chau, H. N. P. Spatial pattern formation in chemical and 11 biological systems. J. Chem. Soc. Faraday Trans. 93, 3601-3610 (1997).

12 5. Reeves, G. T., Muratov, C. B., Schüpbach, T. \& Shvartsman, S. Y. Quantitative Models of 13 Developmental Pattern Formation. Dev. Cell 11, 289-300 (2006).

14 6. Meinhardt, H. Models of biological pattern formation: from elementary steps to the 15 organization of embryonic axes. Curr. Top. Dev. Biol. 81, 1-63 (2008).

16 7. Rietkerk, M. \& van de Koppel, J. Regular pattern formation in real ecosystems. Trends $17 \quad$ Ecol. Evol. 23, 169-175 (2008).

18 8. Kondo, S. \& Miura, T. Reaction-Diffusion Model as a Framework for Understanding 19 Biological Pattern Formation. Science 329, 1616-1620 (2010).

20 9. Lian, X., Wang, H. \& Wang, W. Delay-driven pattern formation in a reaction-diffusion 21 predator-prey model incorporating a prey refuge. J. Stat. Mech. Theory Exp. 2013, $22 \quad$ P04006 (2013).

23 10. Halatek, J. \& Frey, E. Rethinking pattern formation in reaction-diffusion systems. Nat. 24 Phys. 1 (2018). doi:10.1038/s41567-017-0040-5

25 11. Basu, S., Gerchman, Y., Collins, C. H., Arnold, F. H. \& Weiss, R. A synthetic multicellular 26 system for programmed pattern formation. Nature 434, 1130-1134 (2005). 
1 12. Liu, C. et al. Sequential establishment of stripe patterns in an expanding cell population.

$2 \quad$ Science 334, 238-241 (2011).

3 13. Payne, S. et al. Temporal control of self-organized pattern formation without

4 morphogen gradients in bacteria. Mol. Syst. Biol. 9, 697 (2013).

5 14. Cao, Y. et al. Collective Space-Sensing Coordinates Pattern Scaling in Engineered

$6 \quad$ Bacteria. Cell 165, 620-630 (2016).

7 15. Kong, W., Blanchard, A. E., Liao, C. \& Lu, T. Engineering robust and tunable spatial

8 structures with synthetic gene circuits. Nucleic Acids Res. 45, 1005-1014 (2017).

9 16. Gomez, M. M. \& Arcak, M. A tug-of-war mechanism for pattern formation in a genetic

10 network. ACS Synth. Biol. 6, 2056-2066 (2017).

11 17. Borek, B., Hasty, J. \& Tsimring, L. Turing Patterning Using Gene Circuits with Gas-

12 Induced Degradation of Quorum Sensing Molecules. PLOS ONE 11, e0153679 (2016).

13 18. Chen, L. et al. Two-Dimensionality of Yeast Colony Expansion Accompanied by Pattern

$14 \quad$ Formation. PLOS Comput. Biol. 10, e1003979 (2014).

15 19. Diambra, L., Senthivel, V. R., Menendez, D. B. \& Isalan, M. Cooperativity To Increase

16 Turing Pattern Space for Synthetic Biology. ACS Synth. Biol. 4, 177-186 (2015).

17 20. Baker, R. E., Schnell, S. \& Maini, P. K. A clock and wavefront mechanism for somite

18 formation. Dev. Biol. 293, 116-126 (2006).

19 21. Dziekan, P., Nowakowski, B. \& Lemarchand, A. Reaction-diffusion scheme for the clock

20 and wavefront mechanism of pattern formation. Eur. Phys. J. B 87, 77 (2014).

21 22. Cotterell, J., Robert-Moreno, A. \& Sharpe, J. A Local, Self-Organizing Reaction-Diffusion

22 Model Can Explain Somite Patterning in Embryos. Cell Syst. 1, 257-269 (2015).

23 23. Pattern Formation in Morphogenesis: Problems and Mathematical Issues. (Springer-

$24 \quad$ Verlag, 2013). 
1 24. Leppänen, T., Karttunen, M., Barrio, R. A. \& Kaski, K. Morphological transitions and

2 bistability in Turing systems. Phys. Rev. E Stat. Nonlin. Soft Matter Phys. 70, 066202

$3 \quad$ (2004).

4 25. Tuszyński, J. A., Otwinowski, M. \& Dixon, J. M. Spiral-pattern formation and

5 multistability in Landau-Ginzburg systems. Phys. Rev. B 44, 9201-9213 (1991).

6 26. Wu, F., Su, R.-Q., Lai, Y.-C. \& Wang, X. Engineering of a synthetic quadrastable gene

7 network to approach Waddington landscape and cell fate determination. eLife 6,

$8 \quad$ e23702 (2017).

9 27. Tsai, T. Y.-C. et al. Robust, tunable biological oscillations from interlinked positive and 10 negative feedback loops. Science 321, 126-129 (2008).

11 28. Danino, T., Mondragón-Palomino, O., Tsimring, L. \& Hasty, J. A synchronized quorum of 12 genetic clocks. Nature 463, 326-330 (2010).

13 29. Chen, Y., Kim, J. K., Hirning, A. J., Josić, K. \& Bennett, M. R. Emergent genetic oscillations 14 in a synthetic microbial consortium. Science $\mathbf{3 4 9}, 986-989$ (2015).

15 30. Pigolotti, S., Krishna, S. \& Jensen, M. H. Oscillation patterns in negative feedback loops. 16 Proc. Natl. Acad. Sci. 104, 6533-6537 (2007).

17 31. Stricker, J. et al. A fast, robust and tunable synthetic gene oscillator. Nature 456, 516$18519(2008)$.

19 32. Gomez, C. et al. Control of segment number in vertebrate embryos. Nature 454, 335$20 \quad 339(2008)$.

21 33. Gomez, C. \& Pourquié, O. Developmental control of segment numbers in vertebrates. J. 22 Exp. Zoolog. B Mol. Dev. Evol. 312, 533-544 (2009).

23 34. Salazar-ciudad, I., Garcia-fernández, J. \& Solé, R. V. Gene Networks Capable of Pattern 24 Formation: From Induction to Reaction-Diffusion. J. Theor. Biol. 205, 587-603 (2000). 
35. Guantes, R. \& Poyatos, J. F. Multistable Decision Switches for Flexible Control of Epigenetic Differentiation. PLOS Comput. Biol. 4, e1000235 (2008).

3 36. Del Vecchio, D., Abdallah, H., Qian, Y. \& Collins, J. J. A Blueprint for a Synthetic Genetic

$4 \quad$ Feedback Controller to Reprogram Cell Fate. Cell Syst. 4, 109-120.e11 (2017).

5 37. Kicheva, A., Cohen, M. \& Briscoe, J. Developmental pattern formation: insights from physics and biology. Science 338, 210-212 (2012).

38. Scholes, N. S. \& Isalan, M. A three-step framework for programming pattern formation. Curr. Opin. Chem. Biol. 40, 1-7 (2017).

9 39. Azevedo, R. B. R., Lohaus, R., Srinivasan, S., Dang, K. K. \& Burch, C. L. Sexual reproduction 10 selects for robustness and negative epistasis in artificial gene networks. Nature 440, $11 \quad 87-90(2006)$.

40. Perkins, T. J., Jaeger, J., Reinitz, J. \& Glass, L. Reverse Engineering the Gap Gene Network of Drosophila melanogaster. PLOS Comput. Biol. 2, e51 (2006).

41. Kazemian, M. et al. Quantitative Analysis of the Drosophila Segmentation Regulatory Network Using Pattern Generating Potentials. PLOS Biol. 8, e1000456 (2010).

Acknowledgments: We thank Dr. James J Collins for the E. coli K-12 MG1655 strain with $l a c \%$, and Dr. Saeed Tavazoie for lab access for the single-cell microscopy experiments. F.W. was supported by American Heart Association Predoctoral Fellowship 15PRE25710303 (F.W.). This study was financially supported by National Science Foundation Grant DMS1100309 and National Institutes of Health Grant GM106081 (to X.W.), 5R01GM131405-02 (to Y.K.), and by a NIH Director's Early Independence Award to K.R.A. (DP5OD019792).

Author contributions: F.W. and X.W. designed the research. F.W. performed the molecular cloning and patterning experiments. X.F. and K.R.A. designed and performed time-lapse microscopy experiments (agarose pad). T.O., X.C., and Q.Z. participated in the growth 
1 condition experiments. F.W., C.H., J.B., Y.K., and X.W. developed the mathematical modeling

2 and computational analysis. F.W., C.H., F.X., Y.K., K.R.A., and X.W. analyzed the data and

3 wrote the manuscript. X.W., K.R.A., and Y.K. supervised the study.

4

5 Competing interests: There is no conflict of interest.

6

7 Data and materials availability: All the experimental materials and procedures and

8 mathematical models are in the supplementary materials. All other data and code are 9 available from the corresponding author upon reasonable request.

12 Supplementary information

13 Materials and Methods

14 Table S1 - S4

$15 \quad$ Fig. S1 - S7

16 References (1 - 15)

17

18

19 